\title{
Emergency Treatment Process and Evaluation of Teleconference System
}

\author{
AN Zhi-yuan, LI Wen-cui, WANG Chun-ying, ZHANG Yong, LUO Zhen, WU \\ Li-jie
}

Information \& Telecommunication Co. of State Grid Henan Electric Power Company, Zhengzhou 450052, China

xtanzhiyuan@163.com,

Keywords: Teleconference system; Emergency treatment process; Evaluation work.

\begin{abstract}
Teleconference system is an information management system which has functions such as computer control, multimedia technology, network communication and video synthesis technology. The system has been widely used in electric power industry, which raising the efficiency of work. It is very important to ensure safety of the teleconference system. The paper analysis the common faults of teleconference system, and introduces how to deal with emergency and evaluate the work.
\end{abstract}

\section{Introduction}

Information \& telecommunication Co. of State Grid Henan Electric Power Company responsible for the operation maintenance conference system in Henan province, including administration's video conference system of SGCC, emergency conference system of GCC, administration's video conference system of Henan electric power company and emergency conference system of Henan electric power company. The system has three categories, such as MCU meeting, conference terminals and transmission network, about 500 devices.

The provincial conference system currently deploy more sets of different functions, to have more than thousand times meetings every year, involving thousands attending units. Because of the system MCU, terminals, switches and other equipment not included in the real-time monitoring network elements, so when the teleconference system quality becomes poor, lost frames, such as Mosaic phenomenon, it is very hard to judge the reason, mostly rely on experience judgement and speculation [1]. Without warning, at what time and under what circumstances to launch emergency response mechanism is difficult to decide.

MCU can be thought of as a multimedia information exchange, help to realize one point to multipoint communication, video broadcast, audio mixing, accomplish the tandem and switching of each terminal signals[2]. The main models of MCU is huawei 8660 in Henan electric power company. Conference system terminal is an intelligent control equipment, which cooperate with front-end analog camera sampling images, audio, input and output decoding processing after transmission of point to point and point to more intelligent host. The webmaster terminal in Henan province is Huawei 9039s and TE50. Through the transmission network, MCU connected with the terminal meeting equipment. Transmission network include optical transceiver, switches, routers and other information equipment. High-definition conference system currently used video formats for 720 p50hz, display frames of 50 frames per second, video frame rate is 3000 frames $/ \mathrm{min}$. The topology of Henan conference system as shown in the figure below. 


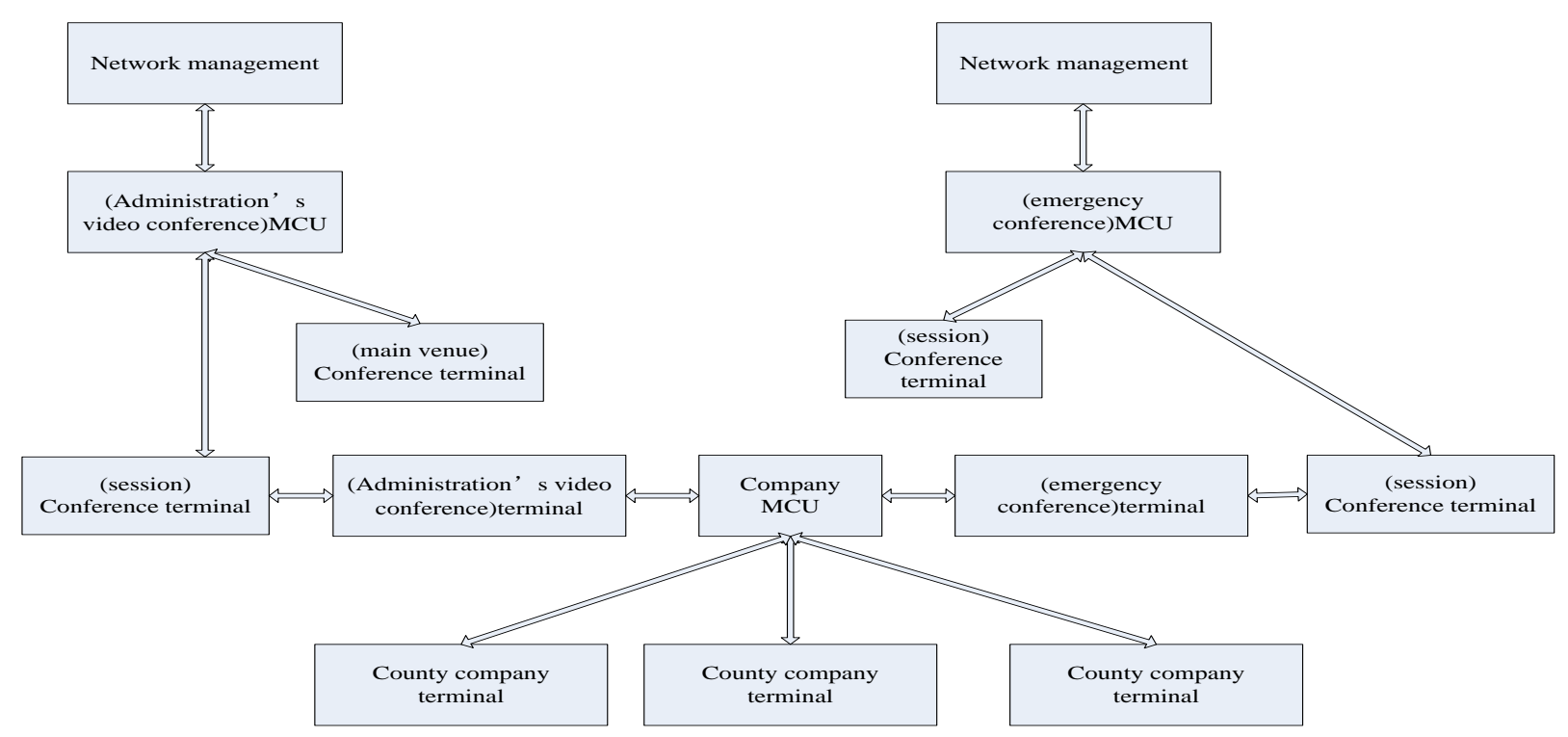

Fig.1 The logical architecture of telecommunication

\section{Common Faults}

Teleconference system common emergency refers to the MCU, conference terminals, peripheral equipment, power supply and transmission channel fault, which caused the images are not fluent or interrupt.

MCU is the core equipment of teleconference system. The MCU equipment failure will affect the whole conference audio and video quality, even lead to its control of the entire conference system paralysis[3]. MCU is a digital processing unit, usually installed in the network node. It is available for multiple sites meeting for communication between each other at the same time, the MCU can be realized in the digital domain audio, video, data and signal and digital signal mixed and switch, but does not affect the quality of the audio and video signals; If the MCU failure, it will affect the normal operation of the whole region TV conference.

Conference terminal is the important equipment in teleconference system, terminal failure will affect the quality in the meeting audio and video signal or lead to interruption of audio and video transmission[4]. Terminal equipment failure relative MCU the smaller effective, generally reflected in: terminal image output, the local voice, terminal software cannot upgrade, camera control failure and unable to remote control remote cameras.

Conference system peripherals mainly includes camera, microphone, audio and video matrix, display devices, etc. Mainly used for local audio and video input and output control. Peripheral equipment failure will affect the quality of audio and video signal or lead to interruption of audio and video transmission.

Power supply is the foundation of the conference system equipment, when the power system failure, it will not be able to hold a meeting.

Transport channels including special E1 channel and IP network. When the meeting transport channel interrupt or error, it will directly affect the quality of the audio and video conference system.

\section{Emergency Organization and Responsibility}

Emergency Organization. (1)According to the communication system of Henan electric power corporation emergency disposal of the emergency plan, when the firm emergency communication system happens, communication operation maintenance units subordinate should be rapidly established an emergency headquarters .

(2)The leadership (or its authorized person) of communication maintenance department should be served as chief of emergency headquarters, and the headquarters should be included all main officials of communication business. 
(3)Emergency headquarters is located in the dispatching department or the appointed places.

(4)Emergency headquarters contains many on-site team members, including communication operation maintenance professional, as well as technical personnel of communication equipment manufacturers.

The Main Responsibilities of the Emergency Headquarters. (1)Emergency headquarters should implement the relevant provisions about how to deal with emergency of Henan electric power company, and perform the emergency treatment process about disposal of large area blackout.

(2)Emergency headquarters should accept emergency disposal of the leading group of emergency instructions, timely start or stop emergency treatment process.

(3)The emergency headquarters is the leadership of emergency disposal work.

(4)Emergency headquarters should declare the state of communication system access or remove the disposal work.

(5)Emergency headquarters should launch or terminate the disposal scheme at the scene.

(6)Emergency headquarters should report and inform related information.

The Main Responsibilities of the On-Site Emergency Team. (1)Emergency team should accept and command the direct leadership of emergency headquarters.

(2)Emergency team should complete the tasks assigned by the emergency headquarters.

(3) Emergency team should formulate the emergency site disposal scheme of communication system, and record the scheme to dispatching communication center of Henan electric power company.

(4)Emergency team should carry out the on-site disposal scheme of the emergency disposal measures, including technical measures, emergency supplies, spare parts, equipment and instruments preparation, to accept the supervision and inspection of the competent department of communications.

(5)According to the incident emergency degree and the running status of communication system, make suggestions about launch or remove the alert, and report to the emergency headquarters.

(6)Emergency team should take effect measures to control the scope of the emergency.

(7)Emergency team should determine the scope of the emergency and timely report to the emergency headquarters.

(8)Emergency team should analysis the major reasons of the communication failure write analysis report and submit it to the emergency headquarters.

\section{Incident report}

(1)The incident report divides into two classes, including emergency report and detailed report. Emergency report refers to the oral report and it is brief. Detailed report submit a detailed report in written form after emergency treatment.

(2)The on-site operation department should be response at the first time and every 20 minutes report the equipment damage and the scope of the emergency to the dispatching department.

(3)The dispatching department should report the equipment damage and the scope of the emergency to the emergency headquarters every 20 minutes, and report significant change immediately

(4)According to the scene disposal situation, the on-site emergency team evaluate the troubleshooting of the time, and report the rate of progress to emergency headquarters every 20 minutes

\section{Evaluation}

After the emergency treatment, the emergency headquarters should organize evaluation:

(1)The on-site emergency team should form a detailed disposal report submitted to the emergency headquarters [5].

(2)The emergency headquarters should analysis the emergency, summed up the scene disposal experience, formulate the measures, and issued [6]. 
(3)The emergency headquarters should supervise the company to improve and perfect the emergency rescue, emergency repair and related measures.

(4)Supervise the company to supple the instruments, materials, spare parts and other supplies reserves immediately.

\section{Announcements}

(1)The related spare parts should be in a good performance. (2)Dealing with emergencies of the teleconference system, such as replace the equipment board, the replacement of the fault card should be repaired in time. If the equipment connection mode is changed, the system should be modified in time to ensure the accuracy of the system diagram. (3)Dealing with emergencies, the relevant departments should coordinate with the logistic support. (4)Disposal personnel should pay attention to comply the rules of the computer room management. (5)Dealing with emergencies, keep in touch with the relevant technical personnel to get technical support.

\section{Conclusion}

Teleconference system connects multiple meeting room by image communication. Various reasons caused the teleconference system failures. It is very hard to judge the reasons, so how to recovery failures in a short period is important to maintainer.

\section{References}

[1] Ookuzu H, Shibata Y. Implementation and Performance Evaluation of a New Teleconference System by Giga Ether Based Omni-directional Video Cameras[C], IEEE International Conference on Distributed Computing Systems Workshops. IEEE Computer Society, 2009:374-379.

[2] Sanaullah A S, Schuckle R W, Pacheco R J. Systems and methods for managing teleconference participant mute state[J]. 2015.

[3] Frankel D P. TELECONFERENCE SYSTEM WITH OVERLAY AUFIO METHOD ASSOCIATE THERETO:, US20150092615[P]. 2015.

[4] Gao J J, Wang Q M, Wang L J, et al. Design and Application of SGCC Main-standby Mode Teleconference System[J]. Electric Power Information \& Communication Technology, 2014.

[5] Saysell E, Routley C. Telemedicine in community-based palliative care: evaluation of a videolink teleconference project.[J]. International Journal of Palliative Nursing, 2003, 9(11):489.

[6] Shibuichi D, Tanaka T, Terashima N, et al. Teleconferencing system using virtual camera[C],Electronic Imaging. International Society for Optics and Photonics, 2000:350-361. 